University of Wollongong

Research Online

Faculty of Science - Papers (Archive)

Faculty of Science, Medicine and Health

$1-1-2012$

\title{
Evidence of animal mtDNA recombination between divergent populations of the potato cyst nematode Globodera pallida
}

Angelique $\mathrm{H}$. Hoolahan

University of Wollongong, angeliqu@uow.edu.au

Vivian C. Blok

James Hutton Institute, UK

Tracey Gibson

University of Wollongong, tgibson@uow.edu.au

Mark Dowton

University of Wollongong, mdowton@uow.edu.au

Follow this and additional works at: https://ro.uow.edu.au/scipapers

Part of the Life Sciences Commons, Physical Sciences and Mathematics Commons, and the Social and Behavioral Sciences Commons

\section{Recommended Citation}

Hoolahan, Angelique H.; Blok, Vivian C.; Gibson, Tracey; and Dowton, Mark: Evidence of animal mtDNA recombination between divergent populations of the potato cyst nematode Globodera pallida 2012, 19-29.

https://ro.uow.edu.au/scipapers/4305

Research Online is the open access institutional repository for the University of Wollongong. For further information contact the UOW Library: research-pubs@uow.edu.au 


\title{
Evidence of animal mtDNA recombination between divergent populations of the potato cyst nematode Globodera pallida
}

\begin{abstract}
Recombination is typically assumed to be absent in animal mitochondrial genomes (mtDNA). However, the maternal mode of inheritance means that recombinant products are indistinguishable from their progenitor molecules. The majority of studies of mtDNA recombination assess past recombination events, where patterns of recombination are inferred by comparing the mtDNA of different individuals. Few studies assess contemporary mtDNA recombination, where recombinant molecules are observed as direct mosaics of known progenitor molecules. Here we use the potato cyst nematode, Globodera pallida, to investigate past and contemporary recombination. Past recombination was assessed within and between populations of $G$. pallida, and contemporary recombination was assessed in the progeny of experimental crosses of these populations. Breeding of genetically divergent organisms may cause paternal mtDNA leakage, resulting in heteroplasmy and facilitating the detection of recombination. To assess contemporary recombination we looked for evidence of recombination between the mtDNA of the parental populations within the mtDNA of progeny. Past recombination was detected between a South American population and several UK populations of G. pallida, as well as between two South American populations. This suggests that these populations may have interbred, paternal mtDNA leakage occurred, and the mtDNA of these populations subsequently recombined. This evidence challenges two dogmas of animal mtDNA evolution; no recombination and maternal inheritance. No contemporary recombination between the parental populations was detected in the progeny of the experimental crosses. This supports current arguments that mtDNA recombination events are rare. More sensitive detection methods may be required to adequately assess contemporary mtDNA recombination in animals.
\end{abstract}

\section{Keywords}

evidence, cyst, animal, nematode, globodera, pallida, mtdna, recombination, between, divergent, populations, potato

\section{Disciplines}

Life Sciences | Physical Sciences and Mathematics | Social and Behavioral Sciences

\section{Publication Details}

Hoolahan, A. H., Blok, V. C., Gibson, T. \& Dowton, M. (2012). Evidence of animal mtDNA recombination between divergent populations of the potato cyst nematode Globodera pallida. Genetica, 140 (1-3), 19-29. 
Evidence of animal mtDNA recombination between divergent populations of the potato cyst nematode

\section{Globodera pallida}

Angelique H. Hoolahan ${ }^{1 *}$, Vivian C. Blok ${ }^{2}$, Tracey Gibson ${ }^{1}$ and Mark Dowton ${ }^{1}$

${ }^{1}$ School of Biological Sciences, University of Wollongong, Wollongong, NSW, 2522, Australia

*Corresponding author; e-mail: angeliqu@uow.edu.au, telephone: +61 24221 3709, fax: +61 242214135

${ }^{2}$ Plant Pathogen Programme, James Hutton Institute, Invergowrie, Dundee, DD25DA, UK

Sequence data from this article have been deposited with the EMBL/GenBank

Data Libraries under accession numbers HQ670242-HQ670401 


\section{ABSTRACT}

Recombination is typically assumed to be absent in animal mitochondrial genomes (mtDNA). However, the maternal mode of inheritance means that recombinant products are indistinguishable from their progenitor molecules. The majority of studies of mtDNA recombination assess past recombination events, where patterns of recombination are inferred by comparing the mtDNA of different individuals. Few studies assess contemporary mtDNA recombination, where recombinant molecules are observed as direct mosaics of known progenitor molecules. Here we use the potato cyst nematode, Globodera pallida, to investigate past and contemporary recombination. Past recombination was assessed within and between populations of G. pallida, and contemporary recombination was assessed in the progeny of experimental crosses of these populations. Breeding of genetically divergent organisms may cause paternal mtDNA leakage, resulting in heteroplasmy and facilitating the detection of recombination. To assess contemporary recombination we looked for evidence of recombination between the mtDNA of the parental populations within the mtDNA of progeny. Past recombination was detected between a South American population and several European populations of $G$. pallida, as well as between two South American populations. This suggests that these populations may have interbred, paternal mtDNA leakage occurred, and the mtDNA of these populations subsequently recombined. This evidence challenges two dogmas of animal mtDNA evolution; no recombination and maternal inheritance. No contemporary recombination between the parental populations was detected in the progeny of the experimental crosses. This supports current arguments that mtDNA recombination events are rare. More sensitive detection methods may be required to adequately assess contemporary mtDNA recombination in animals.

Key words: recombination, mitochondrial DNA, heteroplasmy, paternal leakage; Globodera pallida 


\section{INTRODUCTION}

Animal mitochondrial genomes (mtDNA) are used frequently in phylogenetic analyses. The strictly maternal mode of inheritance (Birky 2008) (except in several bivalves; see Breton et al. 2007) and lack of recombination (Avise 1994; Harrison 1989) make it an ideal marker for tracing evolutionary lineages. However, there is a continuing debate as to the extent of recombination in animal mtDNA (e.g. Hey 2000; Maynard Smith and Smith 2002), and the impact it has on phylogenetics (Kivisild et al. 2000). Strictly maternal inheritance results in identical mtDNA molecules within an individual (i.e. homoplasmy). This makes recombination detection difficult as recombinant products are most readily identified as mosaics of different (heteroplasmic) progenitor molecules (Rokas et al. 2003). Evidence for mtDNA recombination has been reported infrequently in a broad range of animals. Nearly all studies that have looked for evidence of recombination among mitochondrial sequences have looked for evidence of past recombination events - mitochondrial sequences from individuals have been compared, and recombination inferred from the pattern of variation between the sequences (e.g. Armstrong et al. 2007; Shao et al. 2009). Computational programs are frequently used to detect such evidence of recombination in mtDNA sequence data (Awadalla et al. 1999; Ladoukakis and Zouros 2001b; Piganeau et al. 2004; Tsaousis et al. 2005). There have been few studies that have reported evidence of contemporary recombination events. For detection of contemporary recombination, mitochondrial sequences from progeny are directly compared with their parents, and recombination inferred due to an alternating pattern of maternal and paternal sequences in the mitochondrial genome of the progeny. A few studies have directly observed evidence of contemporary recombination (e.g. Kraytsberg et al. 2004; Lunt and Hyman 1997), while other studies have reported evidence of contemporary recombination after several backcrosses (e.g. Arunkumar et al. 2006; Guo et al. 2006). Recombination was also observed in several species of mussels (Burzynski et al. 2003; Ladoukakis and Zouros 2001a). These and other bivalves have two distinct mtDNAs, one inherited maternally, and the other inherited paternally. While females only inherit the maternal mtDNA, males inherit both (Zouros et al. 1994). In several males recombination between parental mtDNA sequences was identified. Simulation studies have attempted to predict the affect that recombination has on phylogenetic analysis. These studies demonstrated that recombination among recently diverged (Posada and Crandall 2002) and rapidly-evolving (Schierup and Hein 2000) sequences can mislead phylogenetic analysis, with distinct incongruence between the resolved phylogeny and the underlying, true phylogeny. However, the impact of recombination on phylogenetics has not been examined using biological data. 
The lack of heteroplasmic mtDNA in animals means that few model systems are available to examine the incidence and mechanism of mtDNA recombination. However, heteroplasmy resulting from paternal mtDNA leakage has been linked with the interbreeding of divergent animal populations or species (Fontaine et al. 2007; Gyllensten et al. 1991; Kaneda et al. 1995; Kondo et al. 1990; Kvist et al. 2003; Magoulas and Zouros 1993; Meusel and Moritz 1993; Niki et al. 1989; Shitara et al. 1998; Zhao et al. 2001). Paternal leakage is suggested to be a result of underdeveloped reproductive barriers between the hybridising organisms that would otherwise prevent paternal mtDNA inheritance (Wagner et al. 1991). The resultant heteroplasmy enables the detection of recombination events between the mtDNAs of the interbred organisms. Consistent with this, the mtDNA of a lizard was described as a mosaic of the mtDNAs from two populations living in close proximity (Ujvari et al. 2007). Further, the mtDNA of a salmon (Ciborowski et al. 2007) and a plant (Jaramillo-Correa and Bousquet 2005) were reported to resemble intermediate forms of the mtDNAs from two organisms living in the same habitat (i.e. that may have interbred). Recombination detected in hybrid zones is typically from past recombination events, as determination of the direct parental sequences is difficult. Therefore, the assessment of contemporary recombination is limited. As yet, no studies have assessed contemporary recombination associated with hybrid zones.

In this study, we looked for evidence of both past and contemporary recombination in the mtDNA of a nematode, Globodera pallida. G. pallida nematodes have a multipartite mitochondrial genome. Most populations have at least 5-6 subgenomic mtDNA circles (scmtDNAs; Armstrong et al. 2000), with an overlapping pattern of mitochondrial genes distributed on the different circles (Gibson et al. 2007). It was previously established that past recombination could be readily detected in one of the subgenomic circles (scmtDNA IV) from one population of G. pallida, P4A (Armstrong et al. 2007). This population was chosen by Armstrong and others, as restriction digestion evidence suggested that its scmtDNA IV had a greater degree of genetic heterogeneity than other populations of this nematode. scmtDNA IV has been reported to be more stable than other subgenomic circles in several population of G. pallida (Armstrong et al. 2007). While scmtDNAs I and III were absent in several populations, scmtDNA IV was detected in all populations studied, using both RFLP and Southern hybridisation approaches. Thus, scmtDNA IV appears to be the most evolutionarily stable of these minicircles, and may be suitable for studying interpopulation genetic interactions. In the present study, we expand on this work by assessing recombination in the mtDNA of other populations of this nematode, demonstrating that this phenomenon is readily detected between a number of populations of G. pallida. 
There are a number of distinct populations of this plant parasitic nematode, with the evolutionary relationships among them relatively well characterised (Madani et al. 2010; Subbotin et al. 2000; Plantard et al. 2008). G. pallida is suggested to have originated in South America (Evans and Stone 1977; Picard et al. 2007) and was imported into Europe and the UK on its host, potato tubers (Stone 1985). The relationships among the five populations of G. pallida used in the present study are shown in Figure 1, with the South American population P4A more closely related to the European and UK populations than to the other South American population, P5A (Subbotin et al. 2000; Grenier et al. 2001). We assessed past and contemporary mtDNA recombination in these populations, and in the progeny of crosses of these populations, respectively, by characterising a $3.4 \mathrm{~kb}$ fragment from one of the six scmtDNAs in the mitochondrial genome of G. pallida. Both the parental populations and the progeny of the crosses were sequenced to investigate whether past and contemporary recombination could be detected, respectively. This has significant implications for animal mtDNA phylogenetic analyses that assume strict maternal inheritance and an absence of recombination as we show that recombination may be occurring when divergent populations interbreed.

\section{METHODS}

Source of the parental G. pallida populations and the progeny from population crosses

To look for evidence of past recombination events within and between populations, populations of the nematode G. pallida were used. Populations were from the potato cyst nematode collection at the James Hutton Institute, which were previously collected from field sites in the UK (Lindley from England; Gourdie and Luffness from Scotland) and South America (P4A, P5A).

To look for evidence of contemporary recombination events, these populations were experimentally crossed and the progeny screened for recombination between the maternal and paternal mtDNA. For the experimental crosses, females from two South American and two UK populations were crossed with the UK population Lindley (see Fig. 1). The experimental crosses of these populations are described in Hoolahan et al. (submitted). To summarise; juveniles from cysts of each population were hatched using a tomato root diffusate (Forrest and Farrer 1983). Juveniles were used to inoculate potato tubers ( $c v$. Désirée). The plants were subsequently washed free of soil and suspended in tanks of aerated water 3 weeks after inoculation of the roots. This process 
facilitated the physical separation of males from females; the adult females, now sedentary, remained attached to the root, while the motile males emerged and fell away from the root. Adult males of the Lindley population were collected for the experimental crosses. Infection of the suspended plants by adult females was assessed after 5 weeks. Protrusions on the surface of the root indicated the presence of adult females, as these are the gravid posterior of the female which later develops into a cyst containing fertilized eggs when the female dies. Plants were suspended for a further 3 weeks to allow any remaining males to emerge before being placed back into the soil. Experimental crosses were then performed by applying approximately 250 males of the Lindley population to each plant infected with females from either population Gourdie, Luffness, P4A or P5A. Cysts from the crossings (containing the progeny) and from the parental populations were recovered from the dried soil of each plant by flotation. Comparable numbers of cysts were recovered from the control plants (parental) and the experimental (progeny) crossings. Each cyst collected from the experimental crossings contains the progeny of a single female. The success of the crosses was assessed via sequencing and RFLP analyses of the nuclear internal transcribed spacer region (ITS) region of the parental populations and progeny nematodes (see Hoolahan et al. submitted). Hybridizations were deemed successful if there were evidence of both maternal and paternal ITS alleles present. Few progeny cysts were collected at the end of each experiment. This may be a due to the females being left in hydroponics for 8 weeks prior to the experimental crosses, as females would typically mate with males several weeks earlier (Chitwood and Buhrer 1945). From each of the GourdieXLindley and LuffnessXLindley cross, 1 cyst was analysed. From the P4AXLindley cross, progeny from 3 cysts were analysed. From the P5AXLindley cross, progeny from 4 cysts were analysed.

\section{Genomic DNA Extraction}

Total genomic DNA was extracted separately from the populations Lindley, Luffness, Gourdie and P5A. (DNA analysis of population P4A was previously reported; Armstrong et al. 2007). For each population, 5 cysts were randomly sampled and pooled for DNA extractions. Genomic DNA from these populations was extracted using the 'salting-out' method of Sunnucks and Hales (1996). DNA was also extracted from the progeny of the experimental crosses. Single cysts collected from the crosses were separately used for genomic DNA extractions with the DNeasy® Blood and Tissue Kit (QIAGEN). 
We looked for evidence of mtDNA recombination by sequencing PCR products of a relatively long (3.4 kb) non-coding mtDNA fragment that were cloned into a vector. This fragment is specific to one of the six small circular mitochondrial genomes (scmtDNA IV) from the mtDNA of G. pallida (Gibson et al. 2007). This region had already been sequenced in 10 clones for the population P4A (Armstrong et al. 2007), and these sequences were retrieved from GenBank (Accession numbers DQ288929-DQ288939). We characterised this fragment in the populations Lindley, Gourdie, Luffness and P5A, and in the progeny of the crosses. Amplifications from extracted genomic DNA used the BIO-X-ACT ${ }^{\mathrm{TM}}$ Long DNA Polymerase Kit (Bioline), and cycling conditions were $94^{\circ} \mathrm{C}$ for $2 \mathrm{~min}, 35-40$ cycles of $94^{\circ} \mathrm{C}$ for $30 \mathrm{~s}, 57^{\circ} \mathrm{C}$ for $30 \mathrm{~s}$ and $68^{\circ} \mathrm{C}$ for $10 \mathrm{~min}$, followed by $68^{\circ} \mathrm{C}$ for 10 min. Products were purified for cloning using either the MinElute® Gel Extraction Kit (QIAGEN) or the Wizard ${ }^{\circledR}$ SV Gel and PCR Clean-Up System (Promega), ligated into the pGEM®-T Easy Vector System (Promega), and transformed into either chemically competent JM109 Escherichia coli cells (Promega) or $\alpha$ Select Electrocompetent cells (Bioline). For sequencing, positive clones were purified using the GeneJET ${ }^{\mathrm{TM}}$ Plasmid Miniprep Kit (Fermentas) or the Wizard® Plus SV Minipreps DNA Purification System (Promega). Sequencing reactions were performed using the BigDye ${ }^{\circledR}$ Terminator v3.1 Cycle Sequencing Kit (Applied Biosystems), and raw sequence data were analysed using ChromasPro (Technelysium Ltd., Tewantin) and BioEdit (Hall 1999).

mtDNA Sequence Alignments and Analysis

Sequences were aligned using MAFFT (multiple sequence alignment based on fast Fourier transform) (Katoh et al. 2002) using the generic mode for globally-alignable sequences (G-INS-I), as this approach was found to be most reliable in a comparison of six popular alignment approaches (Golubchik et al. 2007). The default scoring matrix was used (200PAM). BioEdit (Hall 1999) was used to perform pair-wise alignments and perform sequence identity matricies.

\section{mtDNA Recombination Detection}

Evidence of recombination was assessed using RDP (beta, version 3.41) (Heath et al. 2006; Martin et al. 2010). The settings used were: sequences assumed to be linear, highest acceptable p-value $=0.01$, Bonferroni corrections used (to correct p-values for multiple tests), phylogenetic evidence required, breakpoints polished, check for alignment consistency, list events detected by more than two methods. Methods used were RDP (Martin and Rybicki 2000), GENECONV (Padidam et al. 1999), BOOTSCAN (Martin et al. 2005), Maximum 
Chi-Squared (Smith 1992), CHIMAERA (Posada and Crandall 2001), SiScan (Gibbs et al. 2000), 3SEQ (Boni et al. 2007), TOPAL (McGuire and Wright 2000) and recombination breakpoint hotspots plots (Heath et al. 2006). In addition, highly similar sequences were masked, using the 'automask for optimal recombination detection' feature. Neighbour-joining analysis was performed in RDP using the default settings (Felsenstein 1989). 


\section{RESULTS}

We looked for evidence of both past and contemporary recombination in one of the subgenomic circles (scmtDNA IV) of G. pallida nematodes using different populations of the nematode G. pallida by assessing (1) evidence of past recombination events within other populations of G. pallida, (2) evidence of past recombination events between the various populations of G. pallida, and (3) evidence of contemporary recombination, by performing experimental crosses using males and females from different populations. Recombination was assessed using a range of statistical and phylogenetic methods to determine potential recombinant regions, breakpoints and the parental sequences involved in the recombination event. This analysis approach assesses the statistical support for these potential recombinant regions, constructs the phylogeny of the recombinant and non-recombinant regions of the alignment, and compares the resultant tree topologies. The strongest evidence for recombination is apparent when the topologies of these regions are not congruent. If there were a single underlying hierarchical relationship among the aligned sequences, then it would be expected that phylogenies derived from any region of that alignment would generate the same tree topologies. However, if there has been an exchange of DNA fragments between these sequences, then this will affect the branching order of the effected region, and thus the topology for this region. Therefore, an observation of differing phylogenetic topologies for differing regions in a sequence alignment is indicative of multiple underlying hierarchical relationships, and thus recombination (Husmeier 2005; Husmeier and McGuire 2003). We did not test for recombination between the various circles. Although such intercircle recombination may well occur (the various circles are likely formed by recombination - Gibson et al. 2007), it is difficult to test for this, as the circles that are found in different populations vary (Armstrong et al. 2000).

\section{Evidence of past recombination events}

To look for evidence of ancestral recombination, the $3.4 \mathrm{~kb}$ mtDNA sequences from each population were aligned separately, and each population analysed for evidence of recombination.

\section{$\underline{\text { Recombination is not detectable within four populations of G. pallida }}$}

Within each population, all clones were $>98 \%$ identical for the length of the $3.4 \mathrm{~kb}$ fragment. RDP analysis indicated that none of the four populations sequenced in the present study showed evidence of recombination. We then examined the P4A sequences generated by Armstrong et al. (2007), using RDP. There were eight 
recombination events detected, each with highly significant p-values (Bonferroni corrected p-values between $6 \mathrm{x}$ $10^{-20}$ and $4 \times 10^{-4}$ ). Armstrong et al. (2007) had previously reported evidence of recombination in these sequences using programs that were (TOPAL) and were not (RECPARS and Probabilistic Divergence Measure) applied in the present study. This indicates that the different detection approaches described by Armstrong et al. (2007) and described here were not responsible for the lack of recombination detected in populations Lindley, Gourdie, Luffness and P5A.

Recombination is not detectable between European populations of G. pallida Next we aligned all of the European populations (Gourdie, Lindley and Luffness), and analysed these for evidence of recombination. There was no evidence of recombination between these three populations.

$\underline{\text { Recombination is readily detectable between population P4A and European populations of G. pallida }}$ We then looked for evidence of recombination between P4A and the European populations. To avoid the confounding effects of recombination among the genetically different scmtDNA IV members of the P4A population, we separately assessed each sampled member of the P4A population with the combined European scmtDNA IV sequences from the populations Lindley, Gourdie and Luffness. To do this, we aligned each P4A sequence separately with all of the European population sequences, and analysed each of these 11 alignments for evidence of recombination. These analyses showed strong evidence of recombination between all members of the P4A population and the European populations, except two representatives (DQ288929 and DQ288932) (for a summary, see Table 1; for details of individual statistical analysis outcomes and identified parental sequences, see Online Resource 1). For example, two P4A representatives (DQ288931 and DQ288933) showed strong evidence of the same recombination event, between nucleotides 917 and 1498. Both of these P4A representatives are members of a P4A clade identified by Armstrong et al. (2007), the p22 clade. To illustrate the recombination event further, we describe more fully the recombination evident between DQ288931 and the European populations.

Recombination should be evident where different portions of the alignment confidently support different phylogenies. A neighbour-joining tree of the non-recombinant section (nucleotides 1-916 and 1499-end) showed the topology that would be expected if recombination was absent; the European populations cluster together, while the P4A sequence is the most distantly related sequence (Fig. 2A). A neighbour-joining tree of the 
recombinant section (nucleotides 917-1498) showed a different topology, with the P4A sequence closely related to the Gourdie and Lindley populations (Fig. 2B). This indicates that the recombination event may have involved a section from the Gourdie/Lindley population moving into the P4A sequence. The recombination event could be confirmed by visual inspection of the alignment, particularly if only the variable (informative) sites are included in the alignment (for an example, which involves P4A member DQ288931 and a member from each European population, see Fig. 3). In the non-recombinant section, the European populations are more similar, while the recombinant section indicates a greater similarity between P4A and the Gourdie/Lindley populations. This pattern of similarity was consistent throughout the non-recombinant and recombinant pieces.

A similar, single recombination event was evident when each of DQ288930, DQ288934, DQ288937 and DQ288939 were compared with the European populations. Each of these sequences belongs to clade p37, as identified by Armstrong et al. (2007). The recombinant piece was slightly smaller than in the above example, and there was similarly strong topological evidence in that the recombinant piece clustered with the Gourdie/Lindley populations, whereas the European populations clustered in the non-recombinant piece. A third recombination event was evident when DQ288938 was compared with the European populations - this was also confirmed by topological evidence.

However, in two comparisons, strong evidence of recombination was not directly supported by topological evidence. In the DQ288935/European populations comparison, the non-recombinant piece of DQ288935 clustered with the Gourdie/Lindley populations, while the recombinant piece clustered with the Luffness population. This is consistent with this event being confounded by the earlier recombination of the ancestor to the p22 clade recombining with Gourdie/Lindley, with the secondary recombination being a product of recombination with Luffness.

$\underline{\text { Recombination is detectable between one member of population P4A and P5A }}$

Both P4A and P5A are from South America. We looked for evidence of recombination between P5A and P4A by aligning individual members of P4A with all members of P5A. In contrast to the broad evidence of recombination between members of P4A and the European populations, only a single P4A sequence showed evidence of recombination with the P5A sequences. This was between DQ288934 and the P5A members, where there was evidence of a single recombination event (Table 1). In this alignment, nucleotides 19-1771 showed the 
expected topology, with P4A a monophyletic clade, and the P5A sequence more distantly related, while nucleotides 1772-end had a different topology, with the P5A sequence nesting within the P4A clade. However, the significance of this recombination was lower than for any of the P4A/European recombination events, with a Bonferroni corrected p-value for the former of $2 \times 10^{-5}$.

\section{Evidence of contemporary recombination events}

We then looked for evidence of contemporary mtDNA recombination in a series of experimental crosses. Populations were crossed on a gradient of genetic divergence to assess whether increasing genetic divergence (between parental populations) has an impact on recombination. The mtDNA sequence identity between the parental populations used for each cross were 98\% (Lindley compared with Gourdie; GourdieXLindley), 89\% (Lindley compared with Luffness; LuffnessXLindley), 73-90\% (Lindley compared with P4A; P4AXLindley), and 79\% (Lindley compared with P5A; P5AXLindley), for the $3.4 \mathrm{~kb}$ region analysed. To detect recombination between parental mtDNA, the maternal and paternal mtDNA must be sufficiently different, as recombinants will be most readily detected as mosaics of maternal and paternal sequences. Further, many mitochondrial genomes may have to be sequenced in order to detect the few that are products of recombination. In the present study, we amplified and sequenced a $3.4 \mathrm{~kb}$ fragment of the G. pallida mitochondrial genome to screen for evidence of recombination. We generally sequenced 10 clones from each progeny, producing approximately $34 \mathrm{~kb}$ of sequence data for the assessment. Nevertheless, with $34 \mathrm{~kb}$ we are only able to detect recombination if at least one of the 10 clones is a recombinant.

\section{Ancestral recombination is detectable in the progeny of one cross between European populations of G. pallida}

The populations Lindley, Luffness and Gourdie are all from the UK, and have high sequence similarity, relative to that of the South American populations. For the populations Gourdie and Lindley, sequence data indicated that there were no polymorphic sites between these that could be used to differentiate the maternal and paternal nuclear or mitochondrial DNA in the progeny; comparative sequences from each population were $>98 \%$ identical. Consequently, the success of the hybridization could not be confirmed in this cross. Regardless, RDP analysis did not detect recombination in the mtDNA of the progeny from this cross.

For the cross of populations Luffness and Lindley, 5 clones were $>98 \%$ identical to the maternal mtDNA, while the other 5 clones were only 91\% identical. RDP analysis showed strong evidence of recombination in 4 of the 
latter clones, and weaker evidence of recombination in one of the latter clones. In this case, a central section of the $3.4 \mathrm{~kb}$ fragment showed greater sequence identity with the father than the mother (94\% compared with 75\%), whereas the remaining portion showed greater sequence identity with the mother (93\% compared with 89\%). However, for this 'apparent recombinant' to be a direct product of the experimental cross (i.e. contemporary recombination), we would expect to see portions of sequence that were $99-100 \%$ identical to the maternal and paternal sequences. The observation that the level of sequence identity was lower than this prompted us to consider the possibility that the 'apparent recombinant' was inherited (i.e. was an ancestral polymorphism), rather than derived directly from the experimental cross. Indeed, a comparison of the 'apparent recombinant' with the P4A sequences generated by Armstrong et al. (2007) indicated a high degree of sequence identity (98-99\%) with three of the P4A sequences. This suggests that the progeny sequences were inherited, and that no recombination occurred as a direct result of the cross. To confirm this, we aligned the recombinant and non-recombinant regions to the P4A sequences separately. Both regions were 98-99\% identical to the P4A sequences; c.f. $<95 \%$ for the parental sequences. This further suggests that the recombination was an ancestral event.

Recombination is not detectable in the progeny of a cross between population P4A and a European population of G. pallida

From the P4AXLindley cross, 3 cysts were analysed. The sequence identity matrix shows that the P4AxLindley clones are $99 \%$ identical to at least one of the parental sequences. This suggests that the progeny sequences were inherited, and that no recombination occurred as a direct result of the cross. This was confirmed by RDP analysis identifying the same recombination event in the P4AxLindley progeny and in the parental population P4A.

Recombination is not detectable in the progeny of a cross between population P5A and a European population of G. pallida

From the P5AXLindley cross, 4 cysts were analysed. Almost all clones were >99\% identical within each cyst, and $>98 \%$ identical to either the maternal or paternal mtDNA. In one P5AXLindley cyst, all 10 clones had a 122 bp deletion and an unrelated 148 bp insertion relative to the maternal (P5A) sequence, but were otherwise $>98 \%$ identical to P5A. 


\section{DISCUSSION}

We assessed past and contemporary mtDNA recombination using the nematode G. pallida as a model organism. Past mtDNA recombination has previously been observed in a single G. pallida population (Armstrong et al. 2007). Further, the mtDNA of G. pallida has an overlapping genetic organisation, consistent with the operation of recombination (Gibson et al. 2007). Here we report the first example of evidence of past recombination readily detected between divergent populations of G. pallida. mtDNA sequences from the South American population P4A had recombinant regions that resembled the mtDNA of several European populations. Additionally, we report similar evidence of recombination between the South American populations P4A and P5A. Sequence identity comparisons indicated a discrepancy between the sequences predicted to be involved in a recombination event, and the corresponding sequences in the recombinant. As the recombination events are likely ancestral, it is possible that point mutations and intra- or intermolecular DNA exchanges may have occurred subsequent to the recombination event that was detected.

South American populations of G. pallida are considered to be ancestors of the European populations, with $G$. pallida having originated in Peru (Subbotin et al. 2000; Grenier et al. 2001). The observation of recombination between European-like mtDNA sequences within the mtDNA of P4A is therefore intriguing, and may assist in providing information on the introduction/s of G. pallida into Europe. For example, one explanation for this observation may be that ancestral South American populations of G. pallida contained mtDNA types that resembled the modern European populations Luffness and Lindley/Gourdie. These heterogeneous populations may have (i) interacted with one another to generate the recombinant mtDNA molecules observed within the modern South American population P4A, and (ii) maintained their European-like mtDNA during introductions into Europe, ancestors of the modern populations Luffness, Lindley and Gourdie. In this example, recombination was reported between mtDNA sequences most similar to that observed in the populations Luffness and Lindley, that is, between mtDNA molecules that are approximately 11\% divergent (based on the $3.4 \mathrm{~kb}$ mtDNA region analysed in these populations. Following observations of recombination in the doubly uniparentally inherited mtDNA of mussels, it had previously been suggested that, for homologous recombination to occur, mtDNA molecules ideally need to be approximately $4 \%$ divergent. If DNA sequences are less divergent, then recombination events cannot be detected, and if they are too divergent (for example, >10\%; Smith et al. 2007), then the mismatch repair system may prevent recombination (Ladoukakis and Zouros 
2001a). However, recently recombination between mtDNA molecules that were 16 to 23\% divergent was reported within this mussel system (Ladoukakis et al. 2011). From this observation, Ladoukakis et al. (2011) hypothesised that the mismatch repair system may not be active in the mitochondria of animals, possibly because it is unnecessary in this typically homoplasmic mtDNA. However, it is difficult to compare the results of Ladoukakis et al. (2011) with the homologous recombination observed here, as it is impossible to know the sequence homology between the two ancestral mtDNA molecules that were involved in the past recombination event. Nevertheless, this sequence homology between G. pallida populations can be broadly estimated by calculating the sequence identity between the non-recombinant regions of the modern mtDNA molecules analysed in these populations. This indicated that the sequence divergence ranged from approximately 2 to $24 \%$ between populations identified as having ancestral recombination (including comparisons of the populations P5A and P4A, and P4A and the European populations). These sequence divergence values are equally as high as those reported by Ladoukakis et al. (2011). Thus, these results may also provide evidence that there is no mismatch repair system operating in the mitochondria of those metazoan taxa in which recombination has been detected. Further investigation of this hypothesis is required.

Paternal mtDNA leakage can result in heteroplasmy (Gyllensten et al. 1991), providing the parental molecules necessary for detecting recombination events. Paternal leakage has been observed in experimental (Kondo et al. 1990) and natural (Kvist et al. 2003) crosses of genetically dissimilar organisms. Evidence for animal mtDNA recombination that may have resulted from interbreeding has been reported in a lizard (Ujvari et al. 2007) and a salmon (Ciborowski et al. 2007). In these studies a new mtDNA haplotype was identified that resembled a composite of the mtDNA haplotypes of different organisms living in the same habitat. In the present study we also show evidence of animal mtDNA recombination that may have resulted from interbreeding, through observations of ancestral recombination between divergent populations of G. pallida. This is supported by the observation of paternal mtDNA leakage in experimental crosses of these populations of G. pallida (see Hoolahan et al. submitted).

In the present study, progeny from experimental crosses of these populations of G. pallida had no evidence of contemporary recombination between the mtDNA of the maternal and paternal populations; i.e. resulting directly from the experimental crosses. This is despite several of these progeny having both maternal and paternal mtDNA present (Hoolahan et al. submitted). This supports current arguments that animal mtDNA recombination events are rare (Innan and Nordberg 2002; Kivisild et al. 2000). Recombination may have 
occurred at a frequency below the threshold of detection permitted by the approach used in the present study (i.e. below 10\%). Alternatively, recombination may have occurred in a region of the mtDNA not analysed. This may indicate that more sensitive approaches are may be required to detect contemporary mtDNA recombination. Methods such as high throughput next generation sequencing technologies (NGST) may be suitable (Rokas and Abbot 2009), as a single run of a $3.4 \mathrm{~kb}$ fragment facilitates a detection frequency of $0.003 \%$. Alternatively, a series of experimental backcrosses may assist in amplifying the recombination signal and therefore assist in estimating the biological frequency of recombination. This is because (i) mtDNA haplotypes have an unequal fitness during transmission between generations (de Stordeur 1997), potentially allowing for the accumulation of recombinant mtDNA haplotypes, and (ii)backcrosses may increase the extent of paternal mtDNA leakage, and thus heteroplasmy (Kondo et al. 1990).

Here, we show that animal mtDNA recombination may be occurring between divergent populations. If these conditions are commonly met in natural populations, the assumption that mtDNA does not recombine may be unrealistic. We expect that this assumption may most often be violated when genetically divergent populations breed, as this creates a heteroplasmic mtDNA population, which may facilitate recombination. To assess this, a more comprehensive scale of genetic divergence between hybridizing organisms is needed. Further, the approach for mating these organisms would ideally involve mating males and females individually by performing direct crosses in a microtiter dish, as opposed to crossing the males and females en masse. Finally, for the results to be applicable to all animal mtDNAs, these assessments will ultimately need to be performed in animal models with typical, non-multipartite mitochondrial genomes. 


\section{ACKNOWLEDGEMENTS}

The technical support and assistance of M. Phillips, A. Holt and A. Paterson are gratefully acknowledged. This work was supported by the Australian Research Council [DP0556520]; the University of Wollongong Internationalisation Committee [UIC2007], the Australian Academy of Science [RI 146] and the Scottish Executive’s Rural and Environment Research and Analysis Directorate Workpackage 1.5.4.

\section{DECLARATION}

The authors declare that they have no competing interests.

\section{REFERENCES}

Armstrong MR, Blok VC, Phillips MS (2000) A multipartite mitochondrial genome in the potato cyst nematode Globodera pallida. Genetics 154:181-192

Armstrong MR, Husmeier D, Phillips MS, Blok VC (2007) Segregation and recombination of a multipartite mitochondrial DNA in populations of the potato cyst nematode Globodera pallida. J Mol Evol 64:689-701

Arunkumar KP, Muralidhar M, Nagaraju J (2006) Molecular phylogeny of silkmoths reveals the origin of domesticated silkmoth, Bombyx mori from Chinese Bombyx mandarina and paternal inheritance of Antheraea proylei mitochondrial DNA. Mol Phylogenet Evol 40:419-427

Avise JC (1994) Molecular Markers, Natural History, and Evolution. Chapman \& Hall, New York

Awadalla P, Eyre-Walker A, Smith JM (1999) Linkage disequilibrium and recombination in hominid mitochondrial DNA. Science 286:2524-2525

Birky CW, Jr. (2008) Uniparental inheritance of organelle genes. Curr Biol 18:R692-695

Boni MF, Posada D, Feldman MW (2007) An exact nonparametric method for inferring mosaic structure in sequence triplets. Genetics 176:1035-1047

Breton S, Beaupre HD, Stewart DT, Hoeh WR, Blier PU (2007) The unusual system of doubly uniparental inheritance of mtDNA: Isn't one enough? Trends Genet 23:465474

Burzynski A, Zbawicka M, Skibinski DOF, Wenne R (2003) Evidence for recombination of mtDNA in the marine mussel Mytilus trossulus from the Baltic. Mol Biol Evol 20:388-392

Chitwood BG, Buhrer EM (1945) Summary of soil fumigant tests made against the golden nematode of potatoes (Heterodera rostochiensis, Wollenweber), 1942-1944. Proceedings of the Helminthological Society of Washington 12:39-41

Ciborowski KL, Consuegra S, de Leaniz CG, Beaumont MA, Wang JL, Jordan WC (2007) Rare and fleeting: An example of interspecific recombination in animal mitochondrial DNA. Biol Lett 3:554-557

de Stordeur E (1997) Nonrandom partition of mitochondria in heteroplasmic Drosophila. Heredity 79:615-623

Evans K, Stone AR (1977) A review of the distribution and biology of the potato cystnematodes Globodera rostochiensis and G. pallida. Trop Pest Manage 23:178 - 189 
Felsenstein J (1989) PHYLIP - Phylogeny inference package (version 3.2). Cladistics 5:164166

Fontaine KM, Cooley JR, Simon C (2007) Evidence for paternal leakage in hybrid periodical cicadas (Hemiptera: Magicicada spp.). PLos ONE 2:e892

Forrest JMS, Farrer LA (1983) The response of eggs of the white potato cyst nematode Globodera pallida to diffusate from potato and mustard roots. Ann Appl Biol 103:283-289

Gibbs MJ, Armstrong JS, Gibbs AJ (2000) Sister-Scanning: A Monte Carlo procedure for assessing signals in recombinant sequences. Bioinformatics 16:573-582

Gibson T, Blok VC, Phillips MS, Hong G, Kumarasinghe D, Riley IT, Dowton M (2007) The mitochondrial subgenomes of the nematode Globodera pallida are mosaics: Evidence of recombination in an animal mitochondrial genome. J Mol Evol 64:463-471

Golubchik T, Wise MJ, Easteal S, Jermiin LS (2007) Mind the gaps: Evidence of bias in estimates of multiple sequence alignments. Mol Biol Evol 24:2433-2442

Grenier E, Bossis M, Didier F, Renault L, Didier M (2001) Molecular approaches to the taxonomic position of Peruvian potato cyst nematodes and gene pool similarities in indigenous and imported populations of Globodera. Heredity 86:277-290

Guo X, Liu S, Liu Y (2006) Evidence for recombination of mitochondrial DNA in triploid crucian carp. Genetics 172:1745-1749

Gyllensten U, Wharton D, Josefsson A, Wilson AC (1991) Paternal inheritance of mitochondrial DNA in mice. Nature 352:255-257

Hall TA (1999) BioEdit: A user-friendly biological sequence alignment editor and analysis program for Windows 95/98/NT. Nucl Acid S 41:95-98

Harrison RG (1989) Animal mitochondrial DNA as a genetic marker in population and evolutionary biology. Trends Ecol Evol 4:6-11

Heath L, van der Walt E, Varsani A, Martin DP (2006) Recombination patterns in aphthoviruses mirror those found in other picornaviruses. J Virol 80:11827-11832

Hey J (2000) Human mitochondrial DNA recombination: Can it be true? Trends Ecol Evol 15:181-182

Hoolahan AH, Blok VC, Gibson TG, Dowton M (submitted) Paternal leakage in experimental crosses of populations of the potato cyst nematode Globodera pallida.

Husmeier D (2005) Discriminating between rate heterogeneity and interspecific recombination in DNA sequence alignments with phylogenetic factorial hidden Markov models. Bioinformatics 21(Suppl 2):ii166-ii172

Husmeier D, McGuire G (2003) Detecting recombination in 4-taxa DNA sequence alignments with Bayesian hidden Markov models and Markov chain Monte Carlo. Mol Biol Evol 20:315-337

Innan H, Nordberg M (2002) Recombination or mutational hot spots in human mtDNA? Mol Biol Evol 19:122-1127

Jaramillo-Correa JP, Bousquet J (2005) Mitochondrial genome recombination in the zone of contact between two hybridizing conifers. Genetics 171:1951-1962

Kaneda H, Hayashi J-I, Takahama S, Taya C, Lindahl KF, Yonekawa H (1995) Elimination of paternal mitochondrial DNA in intraspecific crosses during early mouse embryogenesis. Proc Natl Acad Sci USA 92:4542-4546

Katoh K, Misawa K, Kuma K-i, Miyata T (2002) MAFFT: A novel method for rapid multiple sequence alignment based on fast Fourier transform. Nucl Acids Res 30:3059-3066

Kivisild T, Villems R, Jorde LB, Bamshad M, Kumar S, Hedrick P, Dowling T, Stoneking M, Parsons TJ, Irwin JA, Awadalla P, Eyre-Walker A, Smith JM (2000) Questioning evidence for recombination in human mitochondrial DNA. Science 288:1931a 
Kondo R, Satta Y, Matsuura ET, Ishiwa H, Takahata N, Chigusa SI (1990) Incomplete maternal transmission of mitochondrial DNA in Drosophila. Genetics 126:657-663

Kraytsberg Y, Schwartz M, Brown TA, Ebralidse K, Kunz WS, Clayton DA, Vissing J, Khrapko K (2004) Recombination of human mitochondrial DNA. Science 304:981

Kvist L, Martens J, Nazarenko AA, Orell M (2003) Paternal leakage of mitochondrial DNA in the great tit (Parus major). Mol Biol Evol 20:243-247

Ladoukakis ED, Theologidis I, Rodakis GC, Zouros E (2011) Homologous recombination between highly diverged mitochondrial sequences: Examples from maternally and paternally transmitted genomes. Mol Biol Evol 28:1847-1859

Ladoukakis ED, Zouros E (2001a) Direct evidence for homologous recombination in mussel (Mytilus galloprovincialis) mitochondrial DNA. Mol Biol Evol 18:1168-1175

Ladoukakis ED, Zouros E (2001b) Recombination in animal mitochondrial DNA: Evidence from published sequences. Mol Biol Evol 18:2127-2131

Lunt DH, Hyman BC (1997) Animal mitochondrial DNA recombination. Nature 387:247

Madani M, Subbotin SA, Ward LJ, Li X, DeBoer SH (2010) Molecular characterization of Canadian populations of potato cyst nematodes, Globodera rostochiensis and $G$. pallida using ribosomal nuclear RNA and cytochrome $b$ genes. Can J Plant Pathol 32:252-263

Magoulas A, Zouros E (1993) Restriction-site heteroplasmy in anchovy (Engraulis encrasicolus) indicates incidental biparental inheritance of mitochondrial DNA. Mol Biol Evol 10:319-325

Martin D, Rybicki E (2000) RDP: Detection of recombination amongst aligned sequences. Bioinformatics 16:562-563

Martin DP, Lemey P, Lott M, Moulton V, Posada D, Lefeuvre P (2010) RDP3: A flexible and fast computer program for analyzing recombination. Bioinformatics 26:24622463

Martin DP, Posada D, Crandall KA, Williamson C (2005) A modified bootscan algorithm for automated identification of recombinant sequences and recombination breakpoints. AIDS Res Hum Retroviruses 21:98-102

Maynard Smith J, Smith NH (2002) Recombination in animal mitochondrial DNA. Mol Biol Evol 19:2330-2332

McGuire G, Wright F (2000) TOPAL 2.0: Improved detection of mosaic sequences within multiple alignments. Bioinformatics 16:130-134

Meusel MS, Moritz RFA (1993) Transfer of paternal mitochondrial DNA during fertilization of honeybee (Apis mellifera L.) eggs. Curr Genet 24:539-543

Niki Y, Chigusa SI, Matsuura ET (1989) Complete replacement of mitochondrial DNA in Drosophila. Nature 341:551-552

Padidam M, Sawyer S, Fauquet CM (1999) Possible emergence of new geminiviruses by frequent recombination. Virology 265:218-225

Picard D, Sempere T, Plantard O (2007) A northward colonisation of the Andes by the potato cyst nematode during geological times suggests multiple host-shifts from wild to cultivated potatoes. Mol Phylogenet Evol 42:308-316

Piganeau G, Gardner M, Eyre-Walker A (2004) A broad survey of recombination in animal mitochondria. Mol Biol Evol 21:2319-2325

Plantard O, Picard D, Valette S, Scurrah M, Grenier E, Mugniéry D (2008) Origin and genetic diversity of Western European populations of the potato cyst nematode (Globodera pallida) inferred from mitochondrial sequences and microsatellite loci. Mol Ecol 17:2208-2218

Posada D, Crandall KA (2001) Evaluation of methods for detecting recombination from DNA sequences: Computer simulations. Proc Natl Acad Sci USA 98:13757-13762 
Posada D, Crandall KA (2002) The effect of recombination on the accuracy of phylogeny estimation. J Mol Evol 54:396-402

Rokas A, Abbot P (2009) Harnessing genomics for evolutionary insights. Trends Ecol Evol 24:192-200

Rokas A, Ladoukakis E, Zouros E (2003) Animal mitochondrial DNA recombination revisited. Trends Ecol Evol 18:411-417

Schierup MH, Hein J (2000) Recombination and the molecular clock. Mol Biol Evol 17:1578-1579

Shao R, Kirkness EF, Barker SC (2009) The single mitochondrial chromosome typical of animals has evolved into 18 minichromosomes in the human body louse, Pediculus humanus. Genome Res 19:904-912

Shitara H, Hayashi J, Takahama S, Kaneda H, Yonekawa H (1998) Maternal inheritance of mouse mtDNA in interspecific hybrids: Segregation of the leaked paternal mtDNA followed by the prevention of subsequent paternal leakage. Genetics 148:851-857

Smith JM (1992) Analyzing the mosaic structure of genes. J Mol Evol 34:126-129

Stone AR (1985) Co-evolution of potato cyst nematodes and their hosts: Implications for pathotypes and resistance. EPPO Bulletin 15:131-137

Subbotin SA, Halford PD, Warry A, Perry RN (2000) Variations in ribosomal DNA sequences and phylogeny of Globodera parasitising solanaceous plants. Nematology 2:591-604

Sunnucks P, Hales DF (1996) Numerous transposed sequences of mitochondrial cytochrome oxidase I-II in aphids of the genus Sitobion (Hemiptera: Aphididae). Mol Biol Evol 13:510-524

Tsaousis AD, Martin DP, Ladoukakis ED, Posada D, Zouros E (2005) Widespread recombination in published animal mtDNA sequences. Mol Biol Evol 22:925-933

Ujvari B, Dowton M, Madsen T (2007) Mitochondrial DNA recombination in a free-ranging Australian lizard. Biol Lett 3:189-192

Wagner DB, Dong J, Carlson MR, Yanchuk AD (1991) Paternal leakage of mitochondrial DNA in Pinus. Theor Appl Genet 82:510-514

Zhao XB, Chu MX, Li N, Wu CX (2001) Paternal inheritance of mitochondrial DNA in the sheep (Ovine aries). Sci China Ser C 44:321-326

Zouros E, Ball AO, Saavedra C, Freeman KR (1994) An unusual type of mitochondrial DNA inheritance in the blue mussel Mytilus. Proc Natl Acad Sci USA 91:7463-7467 
Table 1. Recombinant regions predicted by RDP analysis of mtDNA sequences from the South American population of G. pallida, P4A, when compared with European populations and population P5A.

\begin{tabular}{|c|c|c|c|c|c|}
\hline P4A clone & $\begin{array}{c}\text { Comparison } \\
\text { mtDNA } \\
\text { population }\end{array}$ & $\begin{array}{c}\text { Number of } \\
\text { recombination } \\
\text { events }\end{array}$ & $\begin{array}{l}\text { Position in } \\
\text { alignment }\end{array}$ & Corrected $p$ & $\begin{array}{l}\text { Topological } \\
\text { evidence }^{\mathrm{a}}\end{array}$ \\
\hline \multicolumn{6}{|l|}{ Clade p22 } \\
\hline DQ288931 & European & 1 & $917-1498$ & $2 \times 10^{-17}$ & Yes \\
\hline DQ288933 & European & 1 & $917-1498$ & $2 \times 10^{-16}$ & Yes \\
\hline DQ288935 & European & 1 & $189-2324$ & $6 \times 10^{-8}$ & No \\
\hline DQ288936 & European & 2 & 155-856, 1513-end & $\underset{11}{1 \times 10^{-11} ; 6 \times 10^{-}}$ & No, No \\
\hline \multicolumn{6}{|l|}{ Clade p37 } \\
\hline DQ288930 & European & 1 & $972-1219$ & $1 \times 10^{-10}$ & Yes \\
\hline \multirow[t]{2}{*}{ DQ288934 } & European & 1 & $972-1219$ & $4 \times 10^{-10}$ & Yes \\
\hline & P5A & 1 & 1772-end & $2 \times 10^{-5}$ & Yes \\
\hline DQ288937 & European & 1 & $972-1269$ & $3 \times 10^{-10}$ & Yes \\
\hline DQ288939 & European & 1 & $972-1219$ & $3 \times 10^{-10}$ & Yes \\
\hline \multicolumn{6}{|l|}{ Clade p15 } \\
\hline DQ288938 & European & 1 & $973-1270$ & $2 \times 10^{-10}$ & Yes \\
\hline
\end{tabular}




\section{FIGURE LEGENDS}

Fig. 1 Relative phylogenetic relationships between populations of Globodera pallida used in this study. Populations Lindley, Gourdie and Luffness are from the UK. Populations P4A and P5A are from South America. For the experimental crosses, males from the population Lindley were crossed with females from the remaining 4 populations. This phylogeny is based on phylogenetic analysis of the internal transcribed spacer region (Grenier et al. 2001; Subbotin et al. 2000)

Fig. 2 Neighbour-Joining phylogenetic analysis of the mtDNA of European populations of G. pallida (Lindley, Gourdie and Luffness) and a representative of the South American population P4A (DQ288931). A comparison of the (A) non-recombinant section, and (B) recombinant section, as identified by RDP analysis. Numbers at nodes indicate bootstrap proportions

Fig. 3 mtDNA sequence alignment of the variable sites between a representative of the European populations of G. pallida (Gourdie, Lindley and Luffness), and a South American population P4A (DQ288931). Recombinant and non-recombinant sections are indicated by shading, and boxed shading, respectively.

Online Resource 1 Recombinant regions predicted by RDP analysis of mtDNA sequences from the South American population of G. pallida, P4A, when compared with European populations and population P5A. Included for each recombination event detected are: the alignment position, $p$ values generated by programs supporting the event, the overall corrected $p$ value, parental sequences, phylogenies of the recombinant and nonrecombinant regions, and the inferred topological evidence for this event 


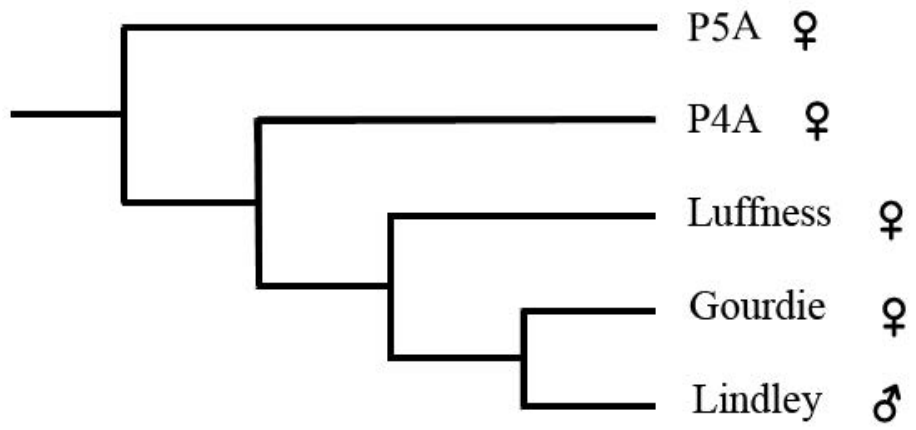


A. Non-recombinant section

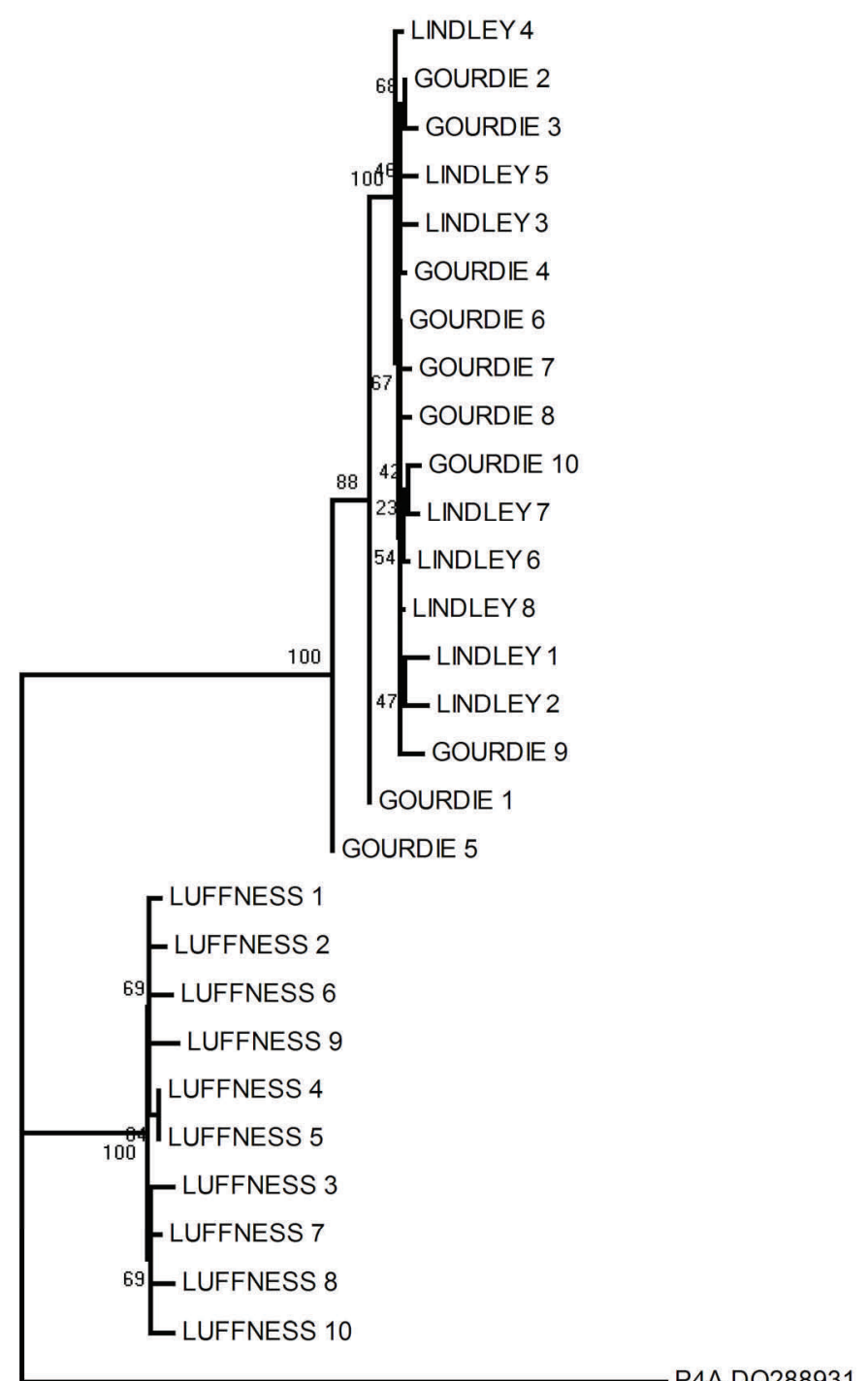

B. Recombinant section

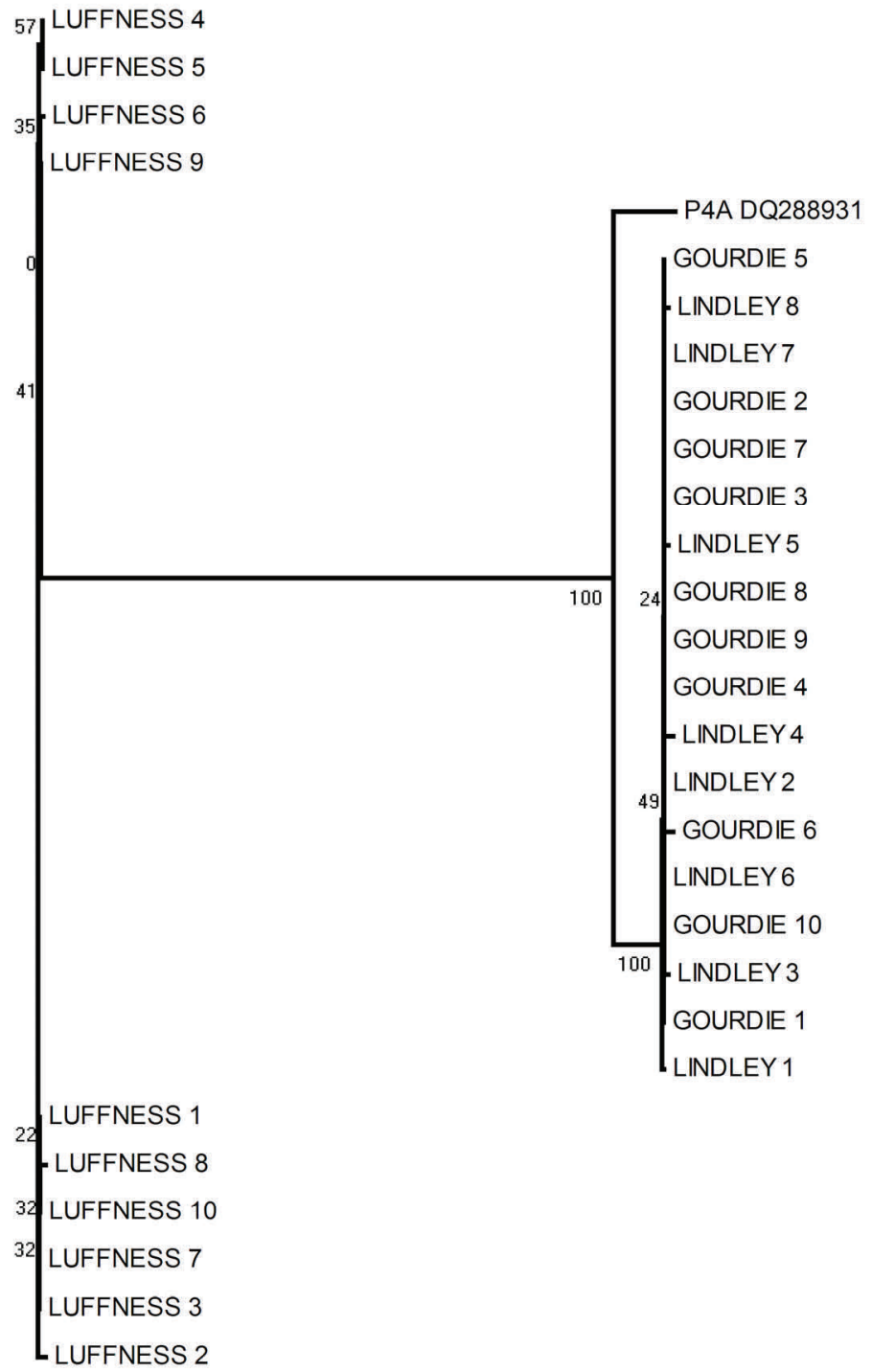


GOURDIE 1 LINDLEY ${ }^{-1}$ LUFFNES $\bar{S} 1$

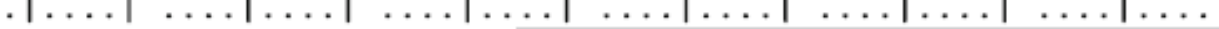
AAGTGGGTCG TTCTGGCCTA CTAGCACTAT GTAGATCAGG CATGAGGTAG TTGGGTGGTA

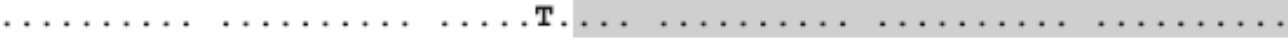
GTA-ATTC.C .A.CA..T. ....T..ATG TAGAT.TTAT TTGTGCAAGA CCATACAACG

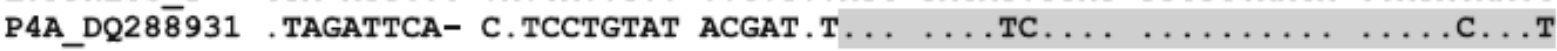

70 80 90 100 110 120

GOURDIE 1 LINDLEY 1 LUFFNES $\bar{S} 1$ P4A DQ288931 $\ldots|\ldots| \ldots|\ldots| \ldots|\ldots| \ldots|\ldots| \ldots|\ldots| \ldots|\ldots| \ldots|\ldots|$ CTGGGAGATC CCGTTGCCGG GCGGTCAGTT TTCTAAGGAG TAAATTGTAA GATTGGCTGT

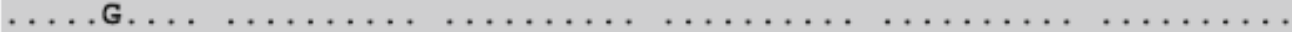
TACAA-TTGT TATAGCTATA CTAT.TCTCC ACTATTTATA ATGTCGAATT AGCC.TTCAC

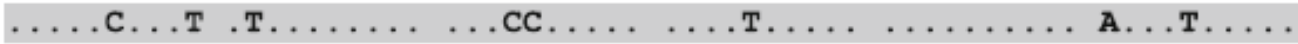

$\begin{array}{rrrrrr}130 & 140 & 150 & 160 & 170 & 180\end{array}$

GOURDIE 1 LINDLEY 1 LUFFNES $\bar{S} 1$

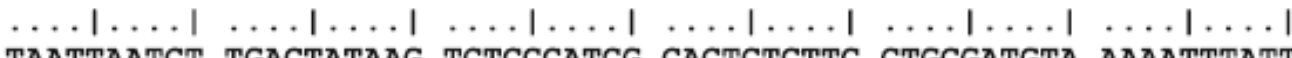
TAATTAATCT TGACTATAAG TCTCCCATCG CACTCTCTTC CTGCGATGTA AAAATTTATT

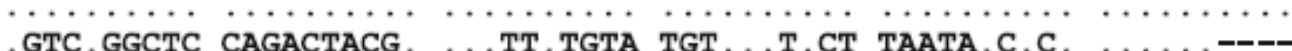
P4A_DQ288931 CG.CAG.C.......... AAC. .T... ... СTC.CGT ---.TTCT.T TTGGCCCTGG

GOURDIE .............. 200 230 240

GOURDIE_-1

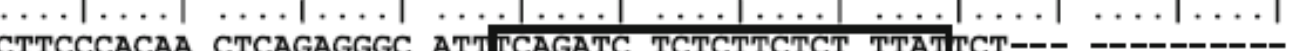
CTTCCACAA CTCAGAGGC ATTPCAGATC TCTCTTCTCT TTATPCT-- LUFFNESS 1

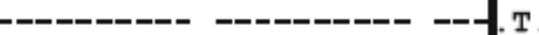
T. . CT. T

\section{-----ACCAC} P4A_DQ28 831 TCGTTTGAGC TCTTTTATTG GACAATATAG CAGGCAAGG CA.A.AATTC GCCTAGGCGT

\section{GOURDIE 1} LINDLEY 1 LUFFNES $\bar{S} 1$ $\ldots|\ldots| \ldots|\ldots| \ldots \mid$ 260 270 280 290 300 СТСТСTTTCT TTСTTTTCTC GTGGATTGGG TTAAAATTCT CCATTATCCC . CCCD ....... . ........ CC G CC ACACT GCT CTCCAT GTTC AT AC

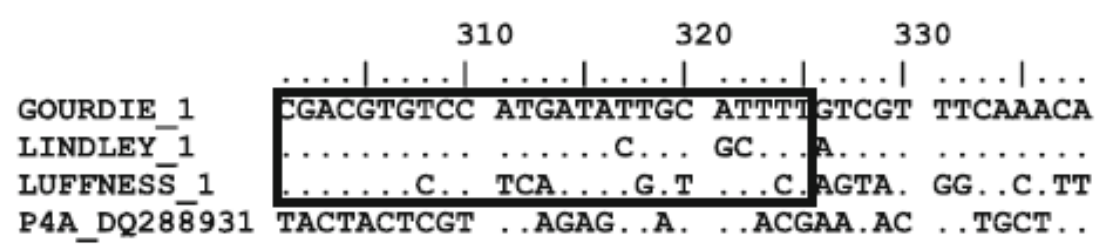

